\title{
Reduced EGFR level potentially mediates the $A \beta 42$-induced neuronal loss in transgenic fruit fly and mouse
}

\section{Dear Editor,}

Memory defect and neuronal loss are both hallmarks of Alzheimer's disease (AD). According to the $\beta$-amyloid $(A \beta)$ hypothesis, persistent accumulation of $A \beta$ peptides, including different aggregation forms, induces neuronal dysfunction and death of neurons. However, one major difference between these two symptoms is that memory defect usually starts in the early stage of AD while neurodegeneration occurs in the advanced stage. Previous studies have reported that the activities of diverse protein factors and their related signaling pathways are associated with the $A \beta$-induced toxicities (Small et al., 2001). But until now, it is still an open question that whether memory defect and neurodegeneration share the same mechanism. Answering this question will be of great help for future drug development and therapy.

We sought to gain insights into the biochemical processes that mediate the $A \beta$ toxicity through in vivo studies of $A \beta 42-$ transgenic fruit flies. Pan-neuronal expression of human $A \beta 42$ in fly brains recapitulates several $A D$-like symptoms such as age-dependent memory loss, accumulation of $A \beta 42$ aggregates and massive neurodegeneration (lijima et al., 2004). Since the APP family proteins or A 342 induce similar pathological phenotypes across a wide range of organisms, from invertebrates to mammals, the molecular basis of $A \beta 42$ toxicity is likely conserved (Gotz et al., 2004). In our previous study, we found a relationship between the EGFR/PI3K pathway and $A \beta 42$-induced memory loss. Accumulation of $A \beta 42$ peptides, especially the oligomers, resulted in an elevation of the
p-EGFR level and a hyperactivation of PI3K activity (Chiang et al., 2010; Wang et al., 2012). These changes caused enhanced long-term depression (LTD) and memory deficits.

Even though feeding A $342-$ express ing flies (lijima et al., 2008) with PI3K inhibitors restored the $A \beta 42$-induced $\mathrm{PI} 3 \mathrm{~K}$ hyperactivity to normal levels and ameliorated memory performance, the neuronal loss was not rescued (Chiang et al., 2010). Similarly, oral administration of gefitinib (Ge), an EGFR inhibitor, to flies also rescued the memory defect (Wang et al., 2012). We then performed HE-staining of paraffin slices with 35-day old fly heads to detect the level of neuronal loss in fly brains. We focused on the mushroom body (MB) region, which is the center for olfactory memory formation. In 35-day old Aß42-expressing fly brains, obvious vacuoles can be found around the $\mathrm{MB}$ somatic region (Fig. 1A). After feeding with $\mathrm{Ge}$, within this $\mathrm{MB}$ region, the ratio of vacuole area to total area was calculated to indicate the extent of neuronal loss. However, similar to the effects of PI3K inhibitors that we found in our previous report, $\mathrm{Ge}(10 \mu \mathrm{g} / \mathrm{mL})$ treatment for 25 days since 10-day old after eclosion did not rescue the loss of neurons (Fig. 1A).

Feeding animals with $\mathrm{Ge}$ could inhibit the level of $p-E G F R$, which usually determines the activity of the EGFR pathway. However, the total protein level of EGFR is also important for maintaining the normal function of this pathway. Thus, we tried to detect the total EGFR level in fly heads by Western blotting. In 10-day old A 442 -expressing flies that already showed memory defect, the total
EGFR level is similar to that of control flies (Fig. 1B, left). In 35-day old flies, we were surprised to find a dramatically reduced EGFR level in A $\beta 42$-expressing fly heads (Fig. 1B, right). Because no antibody was available to detect the Drosophila p-EGFR level simultaneously, it was still not clear either the EGFR level or the p-EGFR level was linked with the lost neurons.

To further confirm that the EGFR level decreased with age, we turned to use the APP/PS1 double transgenic mice with the expression of two mutated AD-linked transgenes: a chimeric human amyloid beta (A4) precursor protein (APPswe) and a "DeltaE9" mutant of human presenilin 1 (Jankowsky et al., 2001). A neuronal loss phenotype was reported in hippocampus of this APP/ PS1 mice as early as $4-5$ months old by means of direct stereological visualization and NeuN immunoreactivity, a marker of neuronal density (Cohen et al., 2009). In our study, hippocampal tissues from 8-month old $\mathrm{Tg}^{+}$mice and age-matched $\mathrm{Tg}^{-}$mice were dissected respectively and subjected to Western blotting. p-EGFR/Actin and EGFR/Actin ratios are used to indicate the relative concentrations of proteins, and p-EGFR/ EGFR ratio is also calculated and shown (Fig. 1C).

As we speculated, the EGFR/Actin ratio was decreased in APP/PS1 mice compared with that of littermate control mice. This data is consistent with our findings in aged $A \beta 42$-expressing flies. Apart from the change of total EGFR level, there was also a significant reduction in $p$-EGFR/Actin ratio in APP/PS1 mice. However, the $p-E G F R / E G F R$ 
A

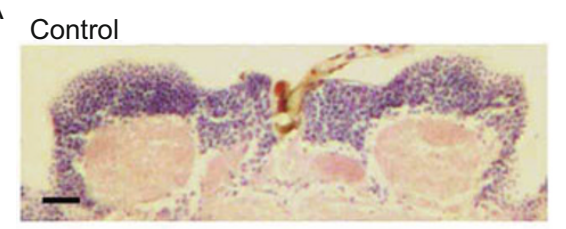

$\mathrm{A} \beta 42$

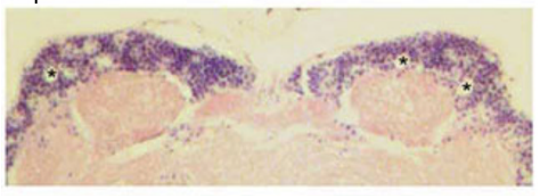

$A \beta 42+\mathrm{Ge} 10$
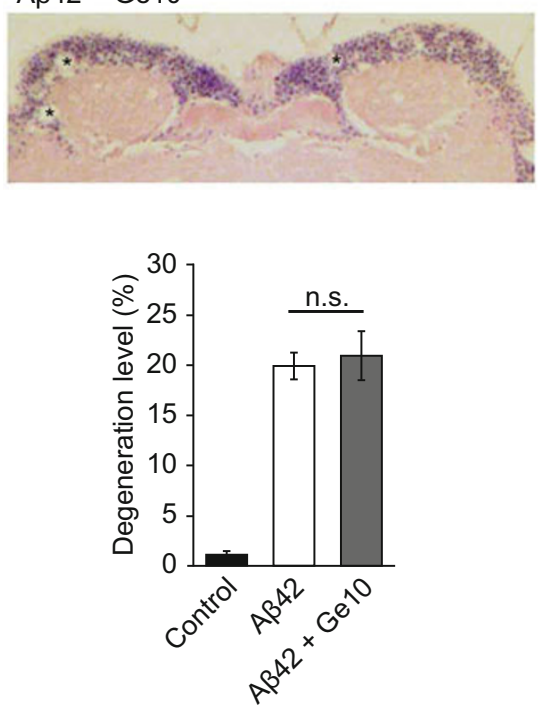

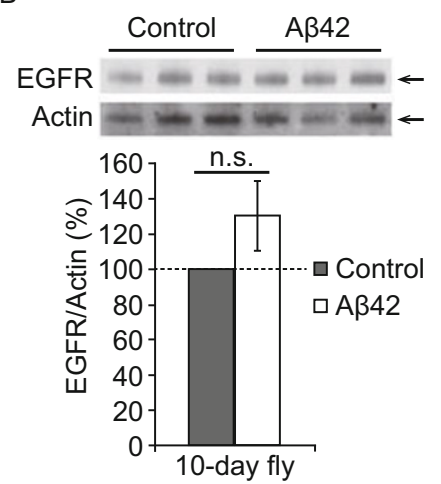

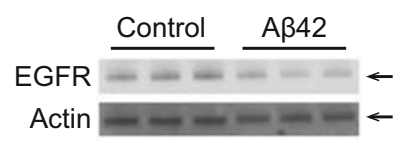

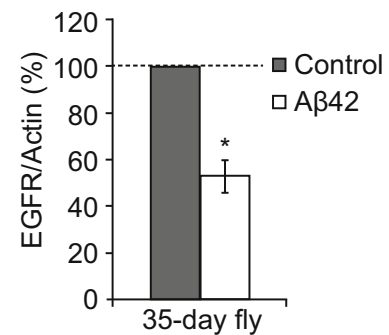

C
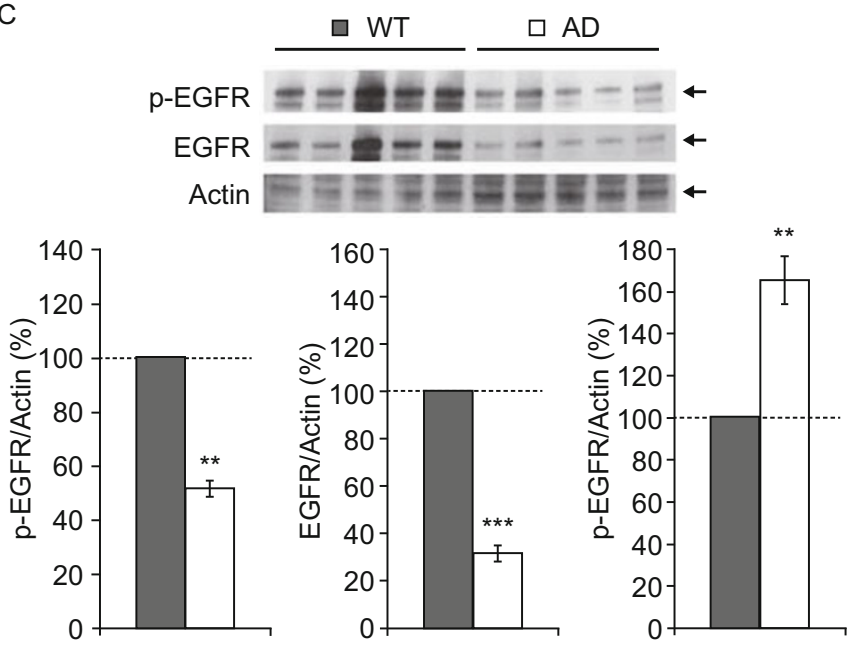

Figure 1. Reduced EGFR level is detected in aged Alzheimer's disease animal models. (A) Representative images of 35-day old fly brain sections with HE staining (up) and statical analysis (down). Human A $\beta 42$ peptides were pan-neuronally expressed under the control of elav-Gal4. Stars indicate the neuronal loss vacuoles in mushroom body somatic region (dark purple) of Aß42-expressing brains (elav-

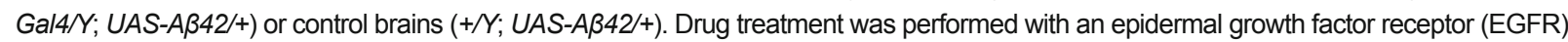
inhibitor, gefitinib (Ge, $10 \mu \mathrm{g} / \mathrm{mL}$ ), since 10-day old for 25 days. $n=14-18$ sections per group. Scale bar: $25 \mu \mathrm{m}$. (B) Representative Western blotting data and statistical results for 10-day old flies (left panel) and 35-day old flies (right panel). EGFR/Actin ratio is used to indicate the total EGFR level. $n=3$ independent experiments. (C) Representative Western blotting data and statistical results of 8-month old APP/ PS1 double transgenic mice and littermate wild type (WT) mice. Protein levels in hippocampus were detected. $n=5$ mice. $t$ test, means \pm SEM, ${ }^{*} P<0.05,{ }^{\star \star} P<0.01,{ }^{* \star *} P<0.001$. n.s., not significant.

ratio was dramatically increased with age. This change could be linked to our behavior tests in mice which found that inhibiting EGFR activation by oral administration of $\mathrm{Ge}$ rescued the memory loss at 8-month old (Wang et al., 2012).

Taking all data together, we raised our working hypothesis that $\mathrm{A} \beta 42$ may have bidirectional functions in regulating the EGFR/PI3K pathway. In the early stage, $A \beta 42$ peptides (especially the oligomers) activate the EGFR and PI3K, influence the normal synaptic plasticity and finally cause memory loss; in the late stage,
$A \beta 42$ peptides induce a reduction of the total EGFR level and cause neuronal loss. Several studies can be considered as support for this hypothesis that cell death depends on the total EGFR level, but not the activation level (Weihua et al., 2008).

EGFR and PI3K are well known for their functions in stimulating cell proliferation and survival, however, accumulating evidences also suggest their biological functions in regulating higher level brain activities, like retrieval and extinction of contextual memory (Chen et al., 2005).
This can help to understand the bidirectional regulation of EGFR by AB42. In younger animals, $A \beta 42$ peptides accumulate extracellularly in the brain and interact with EGFR, possibly through direct binding (Wang et al., 2012). Such interaction consecutively activates the phosphorylation of EGFR and following signaling pathways, including the PI3K pathway, which causes synaptic dysfunction and related memory loss. Additionally, post-mortem studies also support the hypothesis that the activity of AKT (Ser AKT) was increased and 
the activity of PTEN ( $p-P T E N)$ was decreased in AD patients' brains (Rickle et al., 2004, 2006).

In aged animals, accumulated $A \beta 42$ peptides reduce the total EGFR level as we have shown in our study. But the question is how does this happen? At the level of system control, there may exist positive- and negative-feedback regulating mechanisms that maintain the homeostasis of the EGFR family (Citri and Yarden, 2006). As a result, it is quite possible that the consecutive stimulation of EGFR activity by $A \beta 42$ might initiate the negative-feedback loop and thus down-regulate the expression level of EGFR with the aging process.

It should be pointed out that we do not have direct evidence to prove by which means the EGFR level is reduced. However, one report showed that in cultured primary neurons of Tg2576 mice, expression of Swedish APP mutant impaired the normal inactivation, degradation, and ubiquitination of EGFR (Almeida et al., 2006). This result might be regarded as a link between the two directions of regulation on EGFR pathway, and the impaired proteolysis also suggests a subsequent initiation of negative-feedback regulation.

In summary of our findings in young and aged transgenic animals, we propose the hypothesis that the toxic effects of $A \beta 42$ associate with different stages of $A D$ and involve different mechanisms. This may help to enrich our knowledge of this disease and improve the strategy in searching for new drugs.

\section{FOOTNOTES}

L.W. and Y.Z. designed research; L.W. and B.L. performed experiments; L.W. analyzed data and wrote the manuscript. We thank the JoeKai Biotech. LLC (Beijing) for animal experiments and Shannon Zhao for reviewing the manuscript. This work was supported by the National Basic Research Program (973 Program) (Nos. 2009CB941301 and 2013CB835102, to Y.Z.), the Tsinghua University Initiative Scientific Research Program (20111080956, to Y.Z.), and the Fundamental Research Funds for the Central Universities from Beijing University of Chemical Technology (to L.W.)

Lei Wang, Bin Liang and Yi Zhong declare that they have no conflict of interest.

All institutional and national guidelines for the care and use of laboratory animals were followed.

$$
\text { Lei Wang }{ }^{1,2^{*} \bowtie} \text {, Bin Liang }{ }^{2,3^{*}} \text {, Yi Zhong }{ }^{2,4 \bowtie}
$$

${ }^{1}$ College of Life Science and Technology, Beijing University of Chemical Technology, Beijing 100029, China

2 School of Life Sciences, Tsinghua University, Beijing 100084, China

${ }^{3}$ Key Laboratory for Tropical Animal and Plant Ecology of Ministry of Education, College of Life Sciences, Hainan Normal University, Haikou 571158, China

${ }^{4}$ Cold Spring Harbor Laboratory, Cold Spring Harbor, NY 11724, USA

*These authors contributed equally to the work. $\triangle$ Correspondence: zhongyi@cshl.org (Y. Zhong),wanglei@mail.buct.edu.cn (L. Wang)

\section{REFERENCES}

Almeida, C.G., Takahashi, R.H., and Gouras,
G.K. (2006). J Neurosci 26, 4277-4288.

Chen, X., Garelick, M.G., Wang, H., Lil, V., Athos, J., et al. (2005). Nat Neurosci 8, 925-931.

Chiang, H.C., Wang, L., Xie, Z., Yau, A., and Zhong, Y. (2010). Proc Natl Acad Sci U S A 107, 7060-7065.

Citri, A., and Yarden, Y. (2006). Nat Rev Mol Cell Biol 7, 505-516.

Cohen, E., Paulsson, J.F., Blinder, P., Burstyn-Cohen, T., Du, D., et al. (2009). Cell 139, 1157-1169.

Gotz, J., Streffer, J.R., David, D., Schild, A., Hoerndli, F., et al. (2004). Mol Psychiatry 9, 664-683.

Hardy, J., and Selkoe, D.J. (2002). Science 297, 353-356.

lijima, K., Chiang, H.C., Hearn, S.A., Hakker, I., Gatt, A., et al. (2008). PLoS One 3, e1703.

lijima, K., Liu, H.P., Chiang, A.S., Hearn, S.A., Konsolaki, M., et al. (2004). Proc Natl Acad Sci U S A 101, 6623-6628.

Jankowsky, J.L., Slunt, H.H., Ratovitski, T., Jenkins, N.A., Copeland, N.G., et al. (2001). Biomol Eng 17, 157-165.

Rickle, A., Bogdanovic, N., Volkman, I., Winblad, B., Ravid, R., et al. (2004). Neuroreport 15, 955-959.

Rickle, A., Bogdanovic, N., Volkmann, I., Zhou, X., Pei, J.J., et al. (2006). Neurochem Int 48, 114-123.

Small, D.H., Mok, S.S., and Bornstein, J.C. (2001). Nat Rev Neurosci 2, 595-598.

Wang, L., Chiang, H.C., Wu, W., Liang, B., Xie, Z., et al. (2012). Proc Natl Acad Sci U S A 109, 16743-16748.

Weihua, Z., Tsan, R., Huang, W.C., Wu, Q., Chiu, C.H., et al. (2008). Cancer Cell 13, 385-393. 